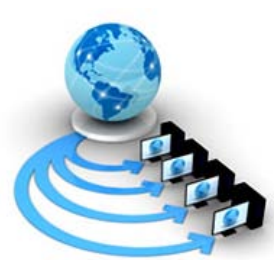

\title{
ENHANCEMENT ON LOW CONTRAST BIRD IMAGES USING IMAGE SIZE DEPENDENT NORMALIZATION TECHNIQUE
}

\author{
Suresha M. \\ Assistant Professor, Department of Computer science \\ Kuvempu University \\ Shimoga, India
}

\author{
Sandeep \\ Research Scholars, Department of Computer science \\ Kuvempu University \\ Shimoga, India
}

\begin{abstract}
A method is proposed for enhancement of low contrast images. Contrast enhancement is a strong influence on contrast ratio to resolve power and detection capability of images. Normalization is one of the common methods for improving contrast digital images and very difficult to improve low contrast image which is the most widely used enhancement processes. Quality of low contrast images is poor due to three reasons. The reasons are camera resolution, light illumination and long distance to capture the images. In this paper, a method image Size Dependent Normalization Technique(ISDNT) is proposed to enhance low contrast bird images to high contrast bird images and enhancement measure in Edge Based Contrast Measurement(EBCM).
\end{abstract}

Keywords: Birds, Contrast Enhancement, EBCM, Image Size Dependent Normalization.

\section{INTRODUCTION}

Image enhancement techniques have been widely used in many applications of image processing where the instinctive quality of images is important for human interpretation. The main objectives of the image processing techniques are to process an image so that the result is more suitable than the original image for specific application [16]. Enhancement techniques contains two broad categories. First, spatial domains techniques are performed to the image plane and they are based on direct manipulation of pixels in an image. Second, frequency domain techniques are straightforward. Simply to compute the fourier transform of the image to be enhanced then multiply the result by a filter and take the inverse transform to produce the enhanced image. The idea of blurring an image by reducing its high frequency components, or sharpening an image by increasing the magnitude of its high frequency components is intuitively easy to understand. However, computationally, it is often more efficient to implement these operations as convolutions by small spatial filters in the spatial domain. Understanding frequency domain concepts is important and leads to enhancement techniques that might not have been thought of by restricting attention to the spatial domain.

The visual appearance of an image is generally characterized by properties brightness and contrast. Brightness refers to the overall intensity level and is therefore influenced by individual gray-level values of all the pixels within an image. Since bright image has more pixel graylevel values closer to the higher end of the intensity scale, it is likely to have a higher average intensity value. Contrast in an image is indicated by the ability of the observer to distinguish separate neighboring parts within an image. This ability to see small details around an individual pixel and larger variations within a neighborhood is provided by the spatial intensity variations of adjacent pixels between two neighboring sub images or within the entire image. Thus, an image may be bright with poor contrast. If the individual target objects in an image have optical characteristics like the background. At the other end of the scale, a dark image may have high contrast if the background is significantly different from the individual objects within the image, or if separate areas within the image have very different reflectance properties. Image enhancement is generally used in the following three cases: noise reduction from image, contrast enhancement of very dark and bright image. Noise reduction is the process of removing noise form a signal or an image. In general, images taken with both digital camera and conventional film cameras will pick up noise from a variety of sources. Therefore, it is required that the noise is removed for many further uses of these images. Contrast enhancement is acquiring clear image through brightness intensity value redistribution. That is, this is enhancing features as stretching interval between dark and brightness area. In this work consider only enhancement on low contrast birds images.

Good contrast images with preserving details are required for many important areas such as remote sensing, machine vision, biomedical image analysis, dynamic and traffic sense analysis, and autonomous navigation. However most of the recorded images suffer from low contrast, which is due to insufficient lighting during image acquiring, wrong setting of shutter speed and aperture size [6]. Thus, contrast enhancement is employed to increase contrast of the image. Enhancement of the images is also useful in edge detection. A good contrast image produces better result for edge detection. An edge detector produces either a set of edge points or edge fragments. Edge detection provides a means of segmenting [5] [7] the image into meaningful regions. Low contrast image is the image whose intensity levels of the pixels resides densely in a narrow range in the histogram of the image. The objects in this type of image are not clear or distinct. To improve the quality of the image and visual perception of human beings, different enhancement methods can be applied. Some methods work in frequency domain, some works in spatial domain and some works in fuzzy domain. For every method, it has some advantages and some disadvantages [14].

Contrast enhancement is a strong influence of contrast ratio on resolving power and detection capability of images. Techniques for improving image contrast are among the most widely used enhancement processes. Using global contrast enhancement, low contrast image can be improved in its 
quality globally. The enhanced output image, with such type of enhancement may not have the noise and ringing artifacts. Local contrast enhancement attempts to increase the appearance of large-scale light-dark transitions [13].

Enhancement can be categorized by two approaches, global and local [2] [4] [11]. Global approaches improve image quality by extending dynamic range of intensity using the histogram of the whole image and local enhancement essentially take into consideration the local properties the image from pixel to pixel. The histogram of the point in the window is calculated and then the histogram equalization transfer function is used to the gray level of the pixel in the center of the mask. This global histogram equalization method is simple and powerful but it cannot adapt to local brightness features of the input image because it uses only global histogram information over the whole image. This fact limits the contrast-stretching ratio in some parts of the image and causes significant contrast losses in the background and other small regions. To overcome this limitation, a local histogram-equalization method has been developed which can also be termed block-overlapped histogram equalization [4] [17]. Here, mainly interested in the contrast enhancement methods. Several methods have been proposed to achieve contrast enhancement invariant to illumination variations. There are called as Gray level transformations, Gamma correction, Contrast stretching, Histogram equalization, Histogram matching [8] [10] [12].

Normalization is one of the common methods used for improving contrast in digital images and Normalization techniques changes range intensity values of the images. Applications include photographs with poor contrast due to glare. Normalization is sometimes called contrast stretching or histogram stretching. The purpose of dynamic range expansion in the various applications is usually to bring the image, or other type of signal, into a range that is more familiar or normal to the senses, hence the term normalization. Often, the motivation is to achieve consistency in dynamic range for a set of data, signals, or images to avoid mental distraction or fatigue. For example, a newspaper will strive to make all the images in an issue share a similar range of grayscale.

Normalization transforms an n-dimensional grayscale image $I:\left\{X \subseteq R^{n}\right\} \rightarrow\{$ Min,...Max $\}$ with intensity values in the range (Min, Max), into a new image $I_{n}:\left\{X \subseteq R^{n}\right\} \rightarrow\{$ Min,...Max $\}$ with intensity values in the range (NewMin, NewMax).

The linear normalization of a grayscale digital image is performed according to the formula:

$$
I_{n}=(I-M i n) \frac{\text { newMax }- \text { newMin }}{\text { Max }- \text { Min }} .
$$

In this paper, low contrast enhancements improve the perceptibility of objects in the scene by enhancing the brightness difference between objects and their backgrounds. So that it works on enhancement on low contrast birds image using image size dependent normalization technique. This method gives fast processing with effective results due to several reasons such as its applied directly to the entire image, not to every pixel of the processed image. It functions in the spatial domain. Over all image size normalization techniques good enhancement on low contrast bird's images.

\section{RELATED WORK}

The related work reveals that enhancement on contrast images techniques widely used several applications. Zohair Al-Ameen et al., [19] proposed easy, fast and reliable technique to improve the contrast of different types of computed tomography (CT) medical images by applying the technique directly to the entire image and normalize it depending on its size in the spatial domain. Shikha Mahajan et al., [16] proposed a survey on various image enhancement techniques. After surveying different technique, it has been concluded that none of the techniques performs efficiently in all fields. W. Cho et al., [18] proposed preprocessing technique that can provide the improved quality of image robust to illumination changes. Authors proposed new adaptive histogram transformation combining histogram equalization and histogram specification. H. Ibrahim et al., [8] proposed a new method known as brightness preserving dynamic histogram equalization (BPDHE), which is an extension to HE that can produce the output image with the mean intensity almost equal to the mean intensity of the input thus fulfill the requirement of maintaining the mean brightness of the image. H. Yoon et al., [10] proposed a new histogram equalization scheme. The conventional global equalization schemes over-equalize these regions so that too bright or dark pixels are resulted and local equalization schemes produce unexpected discontinuities at the boundaries of the blocks. M. Kaur et al., [12] proposed a review of new forms of histogram for image contrast enhancement. The major difference among the methods in this family is the criteria used to divide the input histogram. J. Y. Kim et al., [11] proposed low-pass filter-type mask to get a non-overlapped sub-block histogram-equalization function to produce the high contrast associated with local histogram equalization but with the simplicity of global histogram equalization. This mask also eliminates the blocking effect of nonoverlapped sub-block histogramequalization. C. C. Sun et al., [3] presented a novel contrast enhancement algorithm. The proposed approach enhances the contrast without losing the original histogram characteristics, which is based on the histogram specification technique. It is expected to eliminate the annoying side effects effectively by using the differential information from the input histogram. S.S. Pathak et al., [15] presented Image enhancement to improve the poor quality of an image to make it useful for human and for machine use. This paper suggests methods for contrast image enhancement to preserve brightness of an image. This method is applied on 2D histogram. In 2D histogram preserving color value is very important part. Local feature enhancement technique is used to improve local features of an image. Local feature enhancement technique is used to sharpen the edges. Contrast Stretching with its brightness factor is used to improve the global features of an image. A. Beghdadi et al., [1] proposed edge based contrast measure for image enhancement quality assessment. It is a known fact that human beings are more sensitive to edges. T. K. Kim et al., [17] proposed a block-overlapped histogram equalization system for enhancing contrast of image sequences. The proposed system has various applications such as video door phone, security video cameras, in addition to the original target video camcoders. S. Somorjeet Singh et al., [14] proposed a method to improve the quality of images and it primary concern is the speed or the computational complexity and simplicity using global contrast stretching. H. K. Sawant et al., [9] proposed an image pixel interdependency linear perceptron network (IPILP) for image 
enhancement that provides a better result for contrast enhancement with brightness preservation. IPILP based on curvelet transform and perceptron networks through curvelet image transform into multiresolution mode. Then found the pixel difference for the dependency of contrast. This difference matrix works as a weight vector for perceptron network and the perceptron network is used to adjust the weight of input image or value. G. Sudhavani et al., [6] proposed three enhancement techniques namely fuzzy rule based contrast enhancement, contrast enhancement using intensification (INT) operator, and contrast enhancement using fuzzy expected value (FEV) are presented for the low contrast grayscale images. G. Sudhavani et al., [7] described the application of a modified fuzzy C-means clustering algorithm to the lip segmentation problem. The modified fuzzy C-means algorithm can take the initial membership function from the spatially connected neighboring pixels. G. Sudhavani et al., [5] presents two independent fuzzy based edge linking algorithms which can find a set of edge points in an image and linking these edge points by thresholding. N. Shah et al., [13] proposed a method for enhancement of images. According to authors contrast enhancement is a strong influence of contrast ratio on resolving power and detection capability of images. Techniques for improving image contrast are among the most widely used enhancement processes. Using global contrast enhancement, low contrast image can be improved in its quality globally. The enhanced output image with such type of enhancement may not have the noise and ringing artifacts. Local contrast enhancement attempts to increase the appearance of large-scale light-dark transitions.

\section{PROPOSED METHODOLOGY}

To the best of the author's knowledge, there is no approach for enhancement on low contrast birds image using image size dependent normalization techniques. The algorithm for the proposed methodology is shown in algorithms.

Step1: Input a low contrast image.

Step2: Determine the size of the input image.

Step3: when degraded image divided by the size of the degraded image operation performs after we get enhancement value $\mathrm{K}$.

Step4: Normalization techniques perform with the use of enhancement value $\mathrm{K}$.

Step5: Output image is enhanced(EI).

The quality of low contrast bird's images is poor due to three reasons are is camera resolution, light illumination and long distance to capture images. Here enhancing the low contrast of computed images, two factors to complete the task must be considered, which are speed and efficiency. The proposed technique considers these two factors by supplying a fast processing with effective results. This technique has been utilized in the spatial domain. Moreover, it has been applied to the entire image directly instead of processing the image pixel by pixel. Normalizing image based on its size takes the following course: first, the size of the processed image is determined. Then the enhancement variable $(\mathrm{K})$ is computed Zohair Al-Ameen et al., [19].

$$
K=\frac{\sum_{i=1}^{i} \sum_{j=1}^{j} x(i, j)}{m \times n} .
$$

Where $x(i, j)$ is the low contrast image, the above equation sums all pixel's values and divides them by the size of the image represented by $m$ and $n$. Finally, the image is enhanced using equation (2):

$$
E I=\frac{[x-\min (x)] \times e^{k}}{[\max (x)-\min (x)]} .
$$

Where $x$ is the low contrast image, min and max are the minimum and maximum pixel values of the processed image, $(k)$ is the enhancement variable, and $(E I)$ is the enhanced image.

\section{EXPERIMENTAL RESULTS}

Fig. 1 shows data set for low contrast bird's images enhanced in our paper. Fig. 2 shows enhancement on low contrast birds using image size Dependent Normalization techniques and Enhancement Measure using edge based contrast measurement(EBCM), its check compares original image edge saturation and enhanced image edge saturation. It is well known fact that human beings are more sensitive to edges. It is expected that an enhanced image should have more edge pixels than the original image and this parameter of EBCM does measure the intensity of edge pixels in small windows of the image.

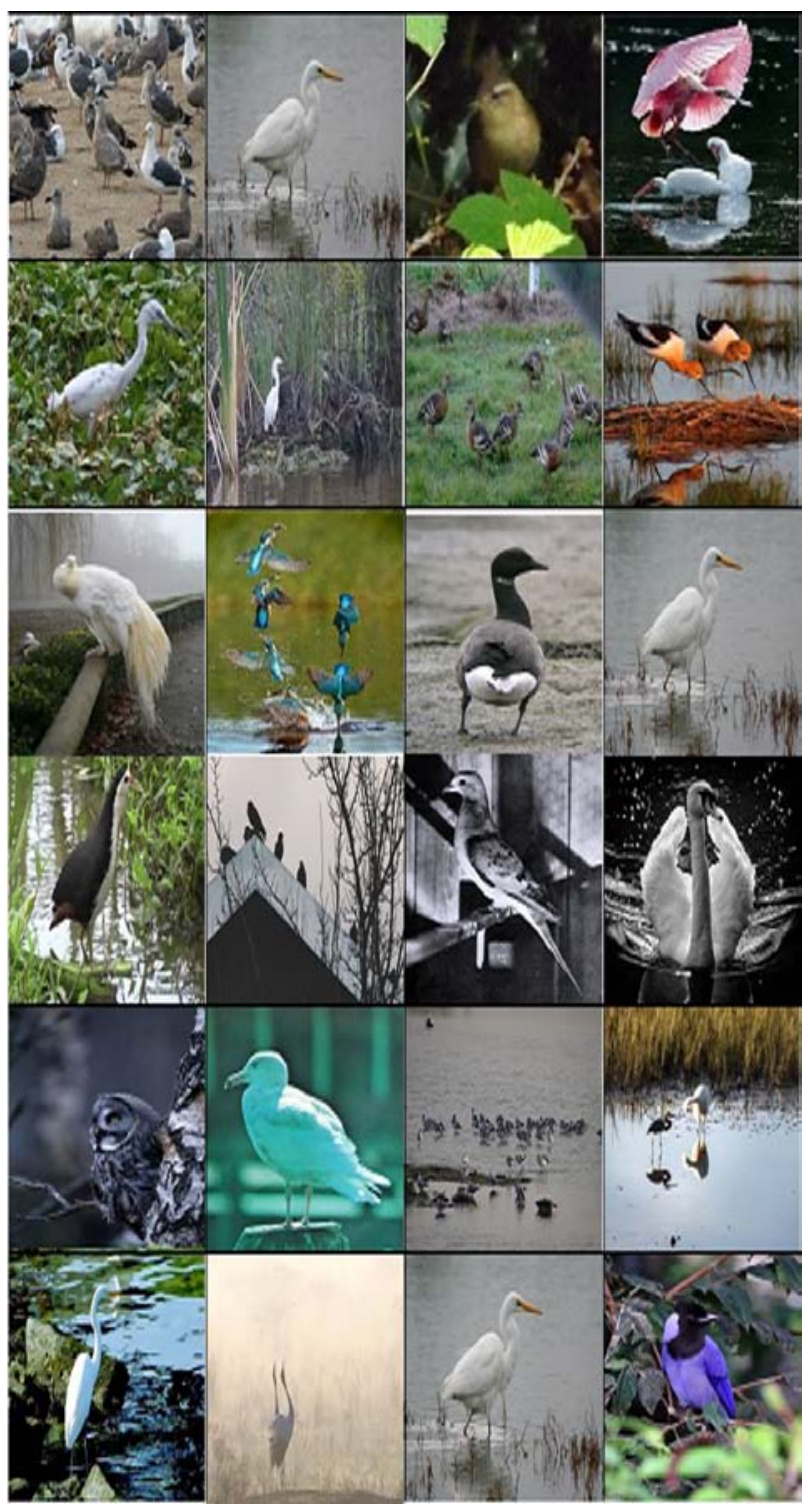

Figure 1: Sample data set of low contrast bird image. 


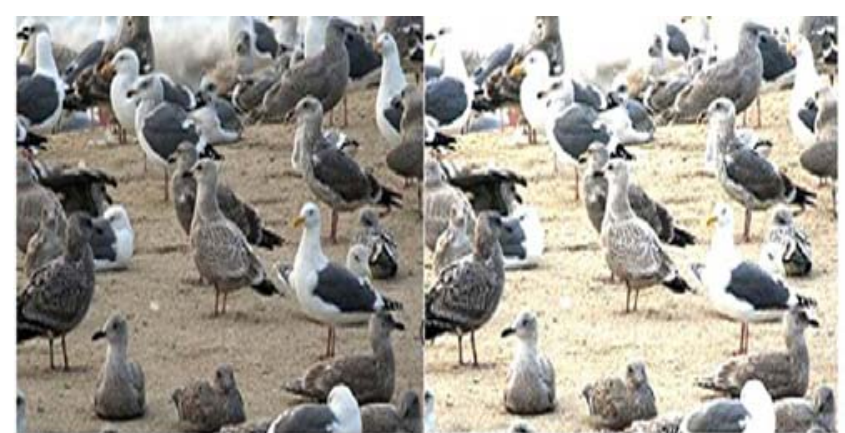

(2) Origind image and EBCSI= $1029 \%$

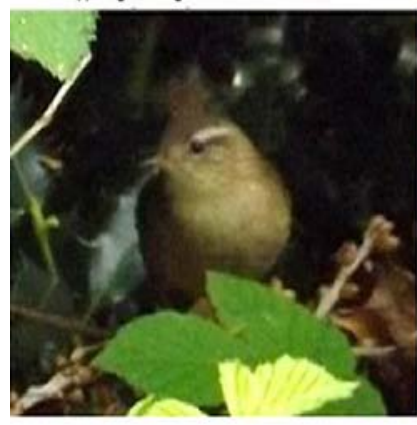

(c) Origital imget and $E B C I=30.0320$

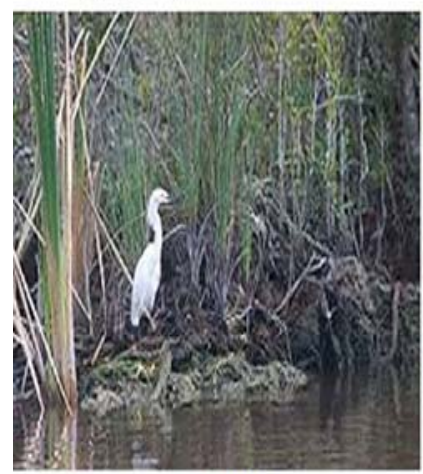

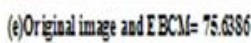

(b) Eabanced image and EBCLI $=1783401$

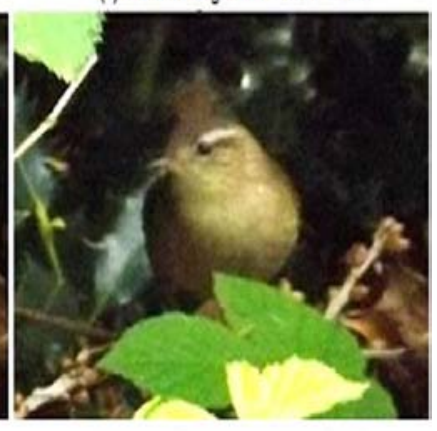

(d) Eabaced imge and EBCUI = Q.658

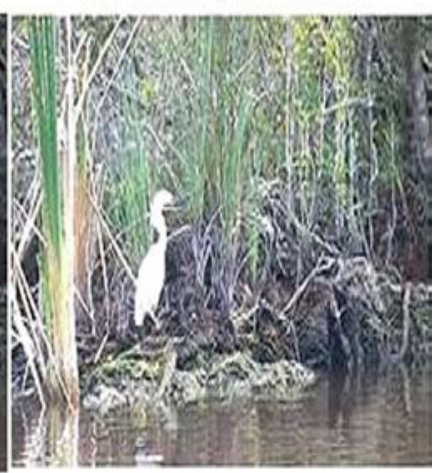

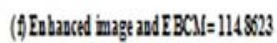

Figure. 2: (a) Column one shows original image with EBCM (Edge Based Contrast Measurement) values (b) Column two shows enhanced image and EBCM values.

\section{CONCLUSION}

The process of enhancement on low contrast birds image using Image Size Dependent Normalization Technique gave extremely good results. When measuring the accuracy with the Edge Based Contrast Measurement found satisfactory results. This method is fast processing with effective results due to several reasons such as its applied directly to the entire image, not to every pixel of the processed image and It functions on the spatial domain. Over all image size normalization techniques gives good enhancement on low contrast bird images.

\section{REFERENCES}

[1] A. Beghdadi and A. L. Negrate, "Contrast enhancement technique based on local detection of edges," Computer Vision Graphics Image Processing., Vol. 46, No. 2, pp. 162174, May 1989.

[2] A. K. Jain, Fundamentals of Digital Image Processing, Prentice-Hall, 1989.

[3] C. C. Sun. S. J. Ruan, M. C. Shie, T. W. Pai, "Dynamic Contrast Enhancement based on Histogram Specification," IEEE Transactions on Consumer Electronics, 51(4), pp. 1300-1305, 2005.
[4] C. Rafael Gonzalez and Richard Eugene woods, Digital image processing pp. 85, 2007.

[5] G. Sudhavani and Dr. K.Satya Prasad, "Segmentation of Lip image by Modified Fuzzy C-means Clustering Algorithm," International Journal of Computer Science \& Network Security, Vol. 9, No. 4, 2009.

[6] G. Sudhavani, M. Srilakshmi, S. Sravani and P. Venkateswara Rao, "K Enhancement of Low Contrast Images Using Fuzzy Techniques,” SPACES, Department of ECE, K L UNIVERSITY, pp. 286-290, 2015.

[7] G. Sudhavani, S. Sravani, P. Venkateswara Rao and K. Satya Prasad, "Fuzzy Edge Linking Process on Fuzzy Noise Filtered Image," International Journal of Computer Applications, Vol. 93, No. 15, 2014.

[8] H. Ibrahim and N. S. P. Kong, "Brightness Preserving Dynamic Histogram Equalization for Image Contrast Enhancement," Consumer Electronics, Vol. 53, No. 4, pp. 1752-1758, 2007.

[9] H. K. Sawant and Mahentra Deore, "A Comprehensive Review of Image Enhancement Techniques,” International Journal of Computer Technology and Electronics Engineering (IJCTEE) Vol.1, Issue. 2, pp. 39-44, 2010.

[10] H. Yoon, Y. Han and H. Hahn, "Image Contrast Enhancement Based Sub-Histogram Equalization Technique without Over-Equalization Noise,” International Journal of Electrical and Electronics Engineering, Vol. 3, No. 6, pp. 323-329, 2009.

[11] J. Y. Kim, L. S. Kim and S. H. Hwang, “An Advanced Contrast Enhancement Using Partially Overlapped SubBlock Histogram Equalization,” IEEE Transactions on Circuits and Systems for Video Technology, Vol. 11, No.4, pp.475-484, 2001.

[12] M. Kaur, J. Kaur and J. Kaur, "Survey of Contrast Enhancement Techniques Based on Histogram Equalization,” International Journal of Advanced Computer Science and Applications, Vol. 2, No. 7, pp. 137-141, 2011.

[13] N. Shah and V. Dahiya, "Comparison of Global - Local Contrast Enhancement in Image Processing,” International Journal of Application or Innovation in Engineering \& Management (IJAIEM), Vol. 4, Issue 11, pp. 16-22, 2015.

[14] S. Somorjeet Singh, Dr. H. Mamata Devi, Th. Tangkeshwar Singh and O. Imocha Singh, "A New Easy Method of Enhancement of Low Contrast Image using Spatial Domain,” International Journal of Computer Applications, Vol. 40 No. 1, pp. 32-34, 2012.

[15] S.S. Pathak and G. Padole, "A Combination of Local and Global Method for Contrast Image Enhancement," International Journal of Computer Science and Network, Vol. 3, Issue. 6, pp. 440-443, 2014.

[16] Shikha Mahajan and Richa Dogra, "A Review on Image Enhancement Techniques,” International Journal of Engineering and Innovative Technology (IJEIT) Volume 4, Issue 11, pp. 108-113, 2015.

[17] T. K. Kim, J. K. Paik, and B. S. Kang, “Contrast enhancement system using spatially adaptive histogram equalization with temporal filtering," IEEE Transaction on Consumer Electronics, Vol. 44, No. 1, pp. 82-86,1998.

[18] Wanhyun Cho, Seongchae Seo, Jinho You and Soonja Kang, "Enhancement Technique of Image Contrast using New Histogram Transformation,” Journal of Computer and Communications, Vol. 2, pp. 52-56 2014.

[19] Zohair Al-Ameen, Ghazali Sulong and Md. Gapar Md. Johar, "Enhancing the Contrast of CT Images by Employing a Novel Image Size Dependent Normalization Techniques," International Journal of Bio-Science and Bio-Technology, Vol. 4, No. 3, pp. 63-68, 2012. 